TEACHING TACTIC

\title{
Maximizing Engagement between Online and On-Campus Students Via Zoom
}

\section{Daniel Orlando Álvarez \\ Pentecostal Theological Seminary}

\section{The context}

My seminary classroom often has as many students online as on-campus. This tactic can be adopted for classroom discussion of most any content, and can be adapted to any class size.

\section{The pedagogical purpose}

The purpose of this exercise was to introduce students to Paul Tillich's Theology of Culture (Oxford University Press, 1980). I wanted to create collegiality between my on-campus and online students by facilitating their engagement with one another. Students would re-think the idea of "religion" they'd been raised with by understanding Tillich's concept of "ultimate concern." The goal was that students would understand how religion, as Tillich uses it, is foundational for all of humanity's existence.

\section{Description of the strategy}

Simultaneous small discussion groups are not possible in a normal Zoom Session, so I used the technology of Zoom ${ }^{1}$ Breakout Rooms to facilitate simultaneous small discussion groups. Before class I logged into Zoom with the host account and set up the breakout room controls. With these controls the host can manually sort participants so that on-campus students can be in mixed groups with online students. I created breakout rooms of three students each. The on-campus students took their own personal devices to quiet places nearby (with WiFi access). As the host, I can enter and leave any breakout room at my discretion to listen to conversations, provide feedback, answer questions, or make suggestions, just as I could if all the small group discussions were physically happening in the classroom. The steps for set up are illustrated in this video ${ }^{2}$ and on this Zoom support page. ${ }^{3}$ This video $^{4}$ shows the breakout rooms in action.

First, groups talked about their notions of religion and defined what this term means for them. Next, they described and defined what "religion" meant for Tillich as compared to what it may mean to them currently. As conversation wound down, I broadcast a message to all groups calling them back to the classroom (for on-campus students) and to the larger Zoom Session (for online students). The students then shared their thoughts and experiences with the larger group.

\section{Why it is effective}

The strategy was effective because the online and on-campus students were able to engage and interact with one another on a closer level. Students carried on more in-depth conversations than they had before. Students took turns describing the emotions and thoughts the readings elicited in them. They listened to one another and considered the thoughts of their peers. I think this exercise was helpful in building collegiality, and for allowing students to "embody" their presence in ways they previously could not.

1 https://zoom.us/ (accessed 14 August 2019).

2 https://www.youtube.com/watch?v=pC_7/GHNH2g.

3 https://support.zoom.us/hc/en-us/articles/206476093-Getting-Started-with-Breakout-Rooms.

4 https://www.youtube.com/watch?v=flJjLtanyxk. 